\title{
Association of CMYC polymorphisms with hepatoblastoma risk
}

\author{
Tianyou Yang ${ }^{1}$, Yang Wen ${ }^{2}$, Jiahao $\mathrm{Li}^{1}$, Tianbao Tan ${ }^{1}$, Jiliang Yang ${ }^{1}$, Jing $\operatorname{Pan}^{1}$, Chao Hu ${ }^{1}$, Yuxiao Yao \\ Jiao Zhang ${ }^{3}$, Suhong $\mathrm{Li}^{4}$, Huimin $\mathrm{Xia}^{1}$, Jing $\mathrm{He}^{1}$, Yan Zou ${ }^{1}$
}

${ }^{1}$ Department of Pediatric Surgery, Guangzhou Women and Children's Medical Center, Guangzhou Medical University, Guangzhou 510623, China; ${ }^{2}$ First Affiliated Hospital of Sun Yat-Sen University, Guangzhou 510080, China; ${ }^{3}$ Department of Pediatric Surgery, The First Affiliated Hospital of Zhengzhou University, Zhengzhou 450052, China; ${ }^{4}$ Department of Pathology, Children Hospital and Women Health Center of Shanxi, Taiyuan 030002, China

Contributions: (I) Conception and design: Y Zou, J He, T Yang, J Li, J Zhang, S Li, H Xia; (II) Administrative support: Y Zou, H Xia; (III) Provision of study materials or patients: T Yang, J Li; (IV) Collection and assembly of data: Y Zou, J He, T Yang, J Li, J Zhang, S Li; (V) Data analysis and interpretation: Y Wen, T Tan, J Yang, J Pan, C Hu, Y Yao; (VI) Manuscript writing: All authors; (VII) Final approval of manuscript: All authors.

Correspondence to: Yan Zou; Jing He. Department of Pediatric Surgery, Guangzhou Women and Children's Medical Center, Guangzhou Medical University, 9 Jinsui Road, Guangzhou 510623, China. Email: 378319696@qq.com, monknut@126.com; hejing198374@gmail.com, hejing@gwcmc.org.

Background: Single-nucleotide polymorphisms (SNPs) in genes may affect gene expression and contribute to cancer susceptibility. This study aimed to explore the association between $C M Y C$ gene polymorphisms and hepatoblastoma risk.

Methods: Hepatoblastoma patients and cancer-free controls were recruited and matched by age and sex. Genotypes were determined by TaqMan, and the strength of the association of interest was determined by calculating odds ratios (ORs) and $95 \%$ confidence intervals (CIs). The distributions of various $C M Y C$ genotypes among subjects were recorded, followed by analyses of associations between $C M Y C$ polymorphisms and hepatoblastoma risk.

Results: A total of 213 hepatoblastoma patients and 958 cancer-free controls were enrolled. No significant associations between the CMYC rs4645943 and rs2070583 polymorphisms and hepatoblastoma risk were found (all $\mathrm{P}>0.05$ ). In stratification analysis based on age, sex, and clinical stage, the CMYC rs4645943 and rs2070583 polymorphisms were not associated with hepatoblastoma susceptibility (all $\mathrm{P}>0.05$ ).

Conclusions: Thus, the CMYC rs4645943 and rs2070583 polymorphisms were not associated with hepatoblastoma risk in the study cohort.

Keywords: $C M Y C$; single-nucleotide polymorphisms (SNPs); hepatoblastoma; cancer susceptibility

Submitted Aug 27, 2019. Accepted for publication Nov 26, 2019.

doi: $10.21037 /$ tcr.2019.12.19

View this article at: http://dx.doi.org/10.21037/tcr.2019.12.19

\section{Introduction}

Hepatoblastoma is the most common hepatic tumor of childhood $(1,2)$. The incidence of hepatoblastoma is about $0.5-1.5$ cases per million, and the mortality rate can be as high as $35-50 \%$ for high-risk patients (3). Over the past decades, efforts have been made to improve the outcome of hepatoblastoma. However, treatment has not changed significantly in the past 20 years (4). In recent years, several unique genetic features have been identified to be associated with hepatoblastoma, providing new insights into the understanding of hepatoblastoma (5). The elucidation of the genetic features of hepatoblastoma is thus of critical importance.

Single-nucleotide polymorphisms (SNPs) are the most common sources of genetic variation in the genome and are frequently associated with potential cancer risk (6). Some SNPs contributing to the progression of hepatoblastoma have been identified. Arai et al. revealed that MDM4 polymorphisms are significantly correlated with the outcomes of hepatoblastoma (7). Based on high-density SNP genotyping microarrays, Suzuki et al. demonstrated 
that expression levels of $I G F 2$ and $H 19$ were significantly correlated with hepatoblastoma (8). c-Myc is a wellknown human transcription factor involved in cell cycle, growth, metabolism, and apoptosis (9). A previous study showed that the $C M Y C$ rs6883267 polymorphism is significantly associated with $C M Y C$ transcription efficiency and poor prognosis in colorectal cancer (10). However, the association between $C M Y C$ polymorphisms and hepatoblastoma remains unclear. This study therefore aimed to investigate the association of $C M Y C$ polymorphisms with hepatoblastoma susceptibility.

\section{Methods}

\section{Patients}

Patients less than 18 years old with a pathologic diagnosis of hepatoblastoma were enrolled. Cancer-free control subjects matched for age and sex were recruited from the same area. All patients and control subjects were genetically unrelated members of the Chinese Han population. Written informed consent was acquired from all participants' legal guardians or parents. The institutional review board of Guangzhou Women and Children's Medical Center approved this study. All patient data were anonymous or de-identified prior to analysis.

\section{CMYC genotyping}

Allelic discrimination of the rs4645943 and rs2070583 polymorphisms of $C M Y C$ was performed using TaqMan reagents (Applied Biosystems, Foster City, CA, USA) according to the manufacturer's protocol, as reported previously (11-14). Control samples of known genotype were also included in each test, including blank, homozygous wild-type, homozygous mutant, and heterozygous samples. Quality control was performed with eight negative and positive control samples on each of the 384-well plates; $10 \%$ of the samples were also randomly selected for a second round of genotyping, and the concordance rate was $100 \%$.

\section{Statistical analysis}

All statistical analyses were performed with SAS software (version 9.1; SAS Institute, Cary, NC, USA). Continuous variables were analyzed using Student's $t$-test or one-way analysis of variance. Categorical variables were analyzed by $\chi^{2}$ test. Differences in allele or genotype frequencies between patients and controls were determined by $\chi^{2}$ test. Hardy-Weinberg equilibrium (HWE) was calculated using a goodness-of-fit $\chi^{2}$ test for biallelic markers. Odds ratios (ORs) and 95\% confidence intervals (CIs) were calculated for evaluation of the strength of the association of interest (15-17). Adjusted ORs were calculated using multivariate analysis after adjusting for age, sex, and clinical stage. Differences were considered significant at $\mathrm{P}<0.05$.

\section{Results}

\section{Characteristics of participants enrolled in this study}

A total of 213 hepatoblastoma patients and 958 control subjects were recruited from Guangdong, Henan, Shaanxi, and Shanxi provinces in China. Males made up the majority of both the hepatoblastoma and control groups, accounting for $60.56 \%$ and $60.44 \%$ of individuals, respectively. Most of the patients had stage II disease $(\mathrm{n}=55)$, followed by stage I $(n=42)$, stage III $(n=40)$, and stage IV $(n=15)$; stage information was lacking for 61 patients (Table S1). There were no significant differences between cases and controls regarding the distributions of age and sex $(\mathrm{P}>0.05$, Table 1$)$.

\section{Association between CMYC polymorphisms and hepatoblastoma risk}

Genotype distributions and associations between $C M Y C$ gene polymorphisms and hepatoblastoma risk are summarized in Table 2. For rs4645943, compared with carriers of the CC genotype, carriers of the CT (OR, 1.10; 95\% CI, 0.81-1.51; $\mathrm{P}=0.532)$ or TT (OR, 1.10; 95\% CI, 0.63-1.92; $\mathrm{P}=0.726)$ genotypes showed no significant associations with hepatoblastoma risk. Moreover, there was no significant association between rs4645943 and hepatoblastoma risk under the additive (OR, 1.07; 95\% CI, $0.85-1.35 ; \mathrm{P}=0.550)$, dominant (OR, 1.10; 95\% CI, 0.82 1.49; $\mathrm{P}=0.512$ ), or recessive models (OR, $1.06 ; 95 \% \mathrm{CI}$, 0.62-1.81; $\mathrm{P}=0.842)$. For rs2070583, compared with carriers of the AA genotype, carriers of the AG (OR, 1.12; 95\% CI, 0.80-1.55; $\mathrm{P}=0.516)$ and GG (OR, 0.84; 95\% CI, 0.35-2.04; $\mathrm{P}=0.699)$ genotypes exhibited no significant associations with hepatoblastoma risk. Similarly, there was no significant association between rs2070583 and hepatoblastoma risk under the additive (OR, 1.04; 95\% CI, 0.79-1.36; $\mathrm{P}=0.783$ ), 
Table 1 Frequency distributions of selected variables in hepatoblastoma patients and controls

\begin{tabular}{lcc}
\hline Variables & Cases $(\mathrm{n}=213), \mathrm{N}(\%)$ & Controls $(\mathrm{n}=958), \mathrm{N}(\%)$ \\
\hline Age range, months & $0.23-149.97$ & $0.004-156.000$ \\
Mean \pm SD & $23.62 \pm 24.36$ & $23.75 \pm 18.30$ \\
$<17$ & $114(53.52)$ & $454(47.39)$ \\
$\geq 17$ & $99(46.48)$ & $504(52.61)$ \\
Sex & & \\
Female & $84(39.44)$ & $379(39.56)$ \\
Male & $129(60.56)$ & $579(60.44)$ \\
Clinical stages & & - \\
I & $42(19.72)$ & - \\
II & $55(25.82)$ & - \\
III & $40(18.78)$ & - \\
IV & $15(7.04)$ & - \\
NA & $61(28.64)$ & - \\
\hline
\end{tabular}

${ }^{\dagger}$, Two-sided $\chi^{2}$ test for distributions between hepatoblastoma patients and cancer-free controls; ${ }^{\ddagger}$, stage information was absent. SD, standard deviation; NA, not applicable.

dominant (OR, 1.08; 95\% CI, 0.79-1.49; $\mathrm{P}=0.618$ ), or recessive models (OR, 0.81; 95\% CI, 0.34-1.97; $\mathrm{P}=0.645)$.

In addition, we found no significant association between hepatoblastoma risk and the combination of the rs 4645943 CT/TT genotype with the rs2070583 AA/AG genotype (OR, 1.13; 95\% CI, 0.84-1.53; $\mathrm{P}=0.410)$.

\section{Stratification analysis of CMYC genotypes and bepatoblastoma risk}

Further analysis showed that neither $C M Y C$ polymorphism was significantly associated with hepatoblastoma risk in any of the subgroups of hepatoblastoma patients (Table 3), which were stratified according to age, sex, and clinical tumor stage (all $\mathrm{P}>0.05$ ). In addition, the combination of the rs4645943 CT/ TT and rs2070583 AA/AG genotypes was not significantly associated with hepatoblastoma risk in any subgroups stratified by age, sex, or clinical tumor stage (all $\mathrm{P}>0.05$ ). These findings suggest that $C M Y C$ polymorphisms are not significantly associated with hepatoblastoma susceptibility.

\section{Discussion}

Our results showed that the $C M Y C$ rs4645943 and rs2070583 polymorphisms were not associated with hepatoblastoma susceptibility. Further stratification analysis based on age, sex, and clinical stage found similar results.

$C M Y C$, encoding the c-Myc protein, is an important oncogene involved in many steps of tumorigenesis, such as proliferation, survival, apoptosis, migration, and invasion (18). A previous study revealed that the expression of c-Myc and cyclin-D1 was significantly elevated in pretreated hepatoblastoma samples but decreased after chemotherapy (19). Myc-expressing mice can present with hepatocellular carcinoma and hepatoblastoma-like tumors, but tumor regression can be induced by inhibiting the expression of Myc (20). Hartwell et al. demonstrated that prolactin suppresses hepatocellular carcinoma by inhibiting the innate immune activation of c-Myc in a mouse model (21). Han et al. found that miR-148a-5p and miR-363-3p negatively regulate the expression of c-Myc to modulate hepatocarcinogenesis (22). These findings suggest that the abnormal expression of $C M Y C$ may play a critical role in the development of liver cancer.

SNPs may be associated with gene transcriptional activity (20,23). For example, CMYC polymorphisms are cis-regulated in the immortalized lymphocytes of HapMap individuals (23). Lee et al. revealed that the CMYC rs4645943 polymorphism was associated with the 


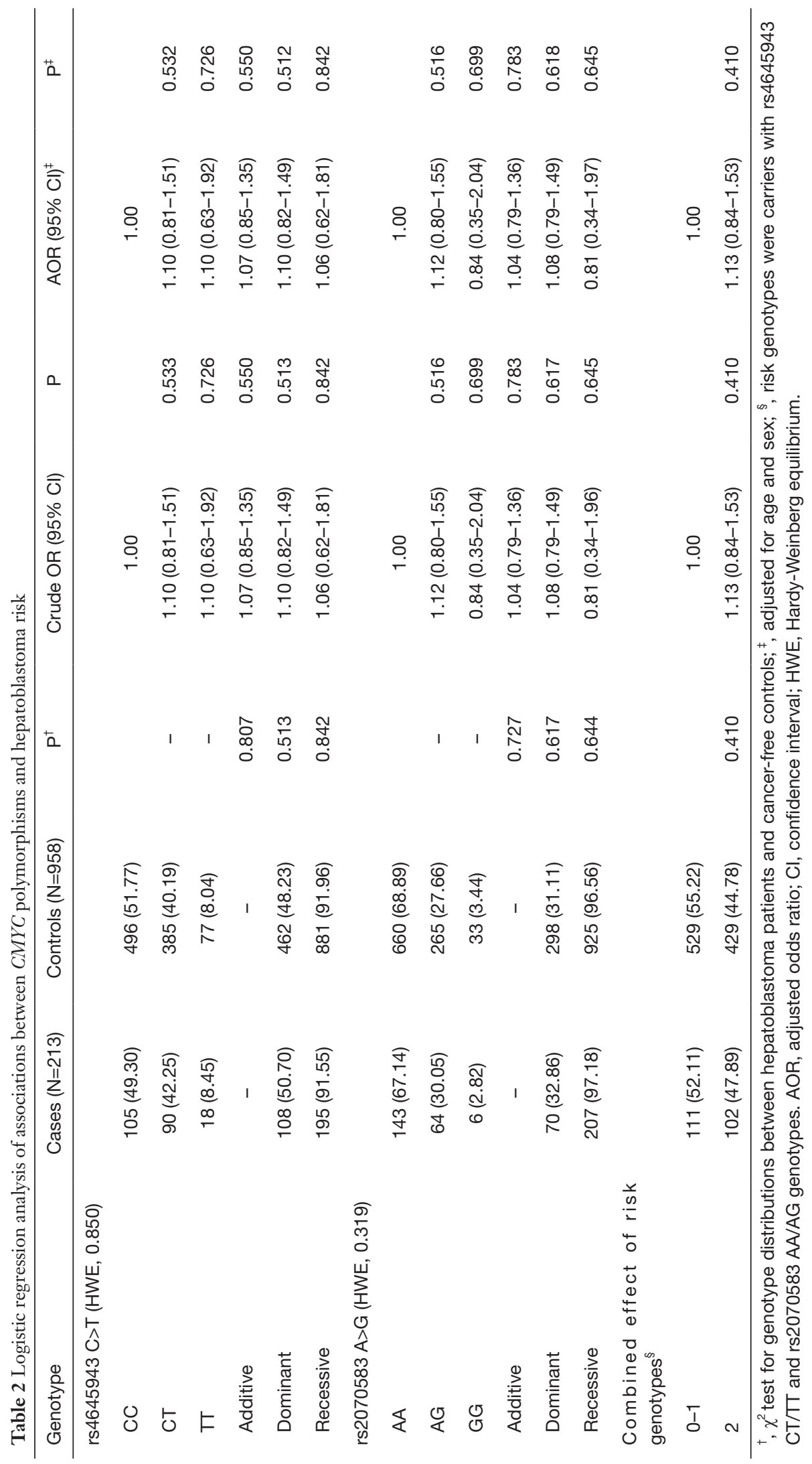




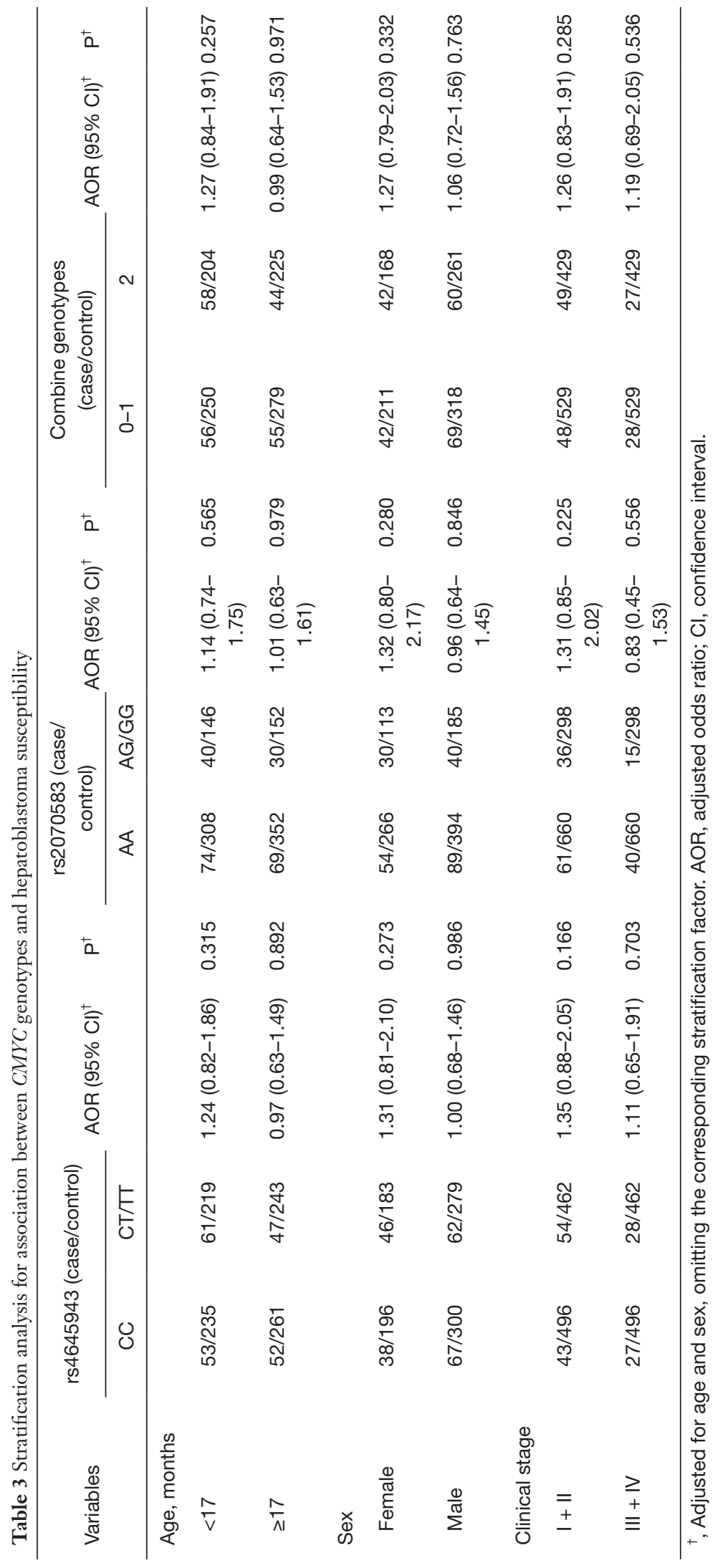


warfarin dose requirement in patients undergoing cardiac valve replacement (24). Moreover, the CMYC rs2070583 polymorphism is significantly associated with coronary heart disease in African Americans (25). However, in the current study, no significant associations were found between the $C M Y C$ rs4645943 and rs2070583 polymorphisms and hepatoblastoma susceptibility in a Han Chinese population. Therefore, we speculate that abnormal expression of $C M Y C$ in hepatoblastoma may not be attributed to $C M Y C$ gene polymorphisms. Wang et al. demonstrated that the role of $C M Y C$ in hepatoblastoma is to impose mutually dependent alterations in gene expression and metabolic re-programming that are not obtained in non-transformed cells and that cooperate to promote tumor growth (26). The activation of $\beta$-catenin is one of the hallmarks of hepatoblastoma, inducing its translocation to the nucleus and activating target genes, including $C M Y C, M M P$ genes, and $V E G F$ to regulate cell proliferation, invasion, and angiogenesis (27). As a member of the Wnt signaling pathway, Wnt ligand binding suppresses the phosphorylation of $\beta$-catenin to inhibit its downstream target genes, such as $C M Y C$, repressing cell proliferation (28). Taken together, this evidence suggests that the abnormal expression of $C M Y C$ (or the protein c-Myc) in hepatoblastoma may largely depend on the regulation of upstream effectors, rather than its genetically encoded information.

Some limitations of this study should be mentioned. First, although we tried to recruit a large number of hepatoblastoma patients, the sample size in this study was still relatively small, and more patients are required to further validate our findings. Second, due to a lack of detailed information on the patients, associations between $C M Y C$ polymorphisms and clinical characteristics, such as tumor size and lymph node metastasis, were not analyzed in this study. Lastly, the study population does not represent the complete Chinese population.

\section{Conclusions}

In summary, the $C M Y C$ rs4645943 and rs2070583 polymorphisms may not be associated with hepatoblastoma risk. The abnormal regulation of $C M Y C$ in hepatoblastoma may therefore require further investigations and explanation.

\section{Acknowledgments}

Funding: This research was funded by the National Natural Science Foundation of China, grant numbers 81602199 and 81802333; the Guangzhou Science Technology and Innovation Commission, grant number 201607010395; the Natural Science Foundation of Guangdong Province, China, grant numbers 2016A030313496 and 2018A030310053. The funders had no role in the design of the study; in the collection, analyses, or interpretation of data; in the writing of the manuscript, or in the decision to publish the results.

\section{Footnote}

Conflicts of Interest: The authors have completed the ICMJE uniform disclosure form (available at http://dx.doi. org/10.21037/tcr.2019.12.19). The authors have no conflicts of interest to declare.

Ethical Statement: The authors are accountable for all aspects of the work in ensuring that questions related to the accuracy or integrity of any part of the work are appropriately investigated and resolved. The study was conducted in accordance with the Declaration of Helsinki (as revised in 2013). Written informed consent was acquired from all participants' legal guardians or parents. The institutional review board of Guangzhou Women and Children's Medical Center approved this study (No. 2017120101). All patient data were anonymous or deidentified prior to analysis.

Open Access Statement: This is an Open Access article distributed in accordance with the Creative Commons Attribution-NonCommercial-NoDerivs 4.0 International License (CC BY-NC-ND 4.0), which permits the noncommercial replication and distribution of the article with the strict proviso that no changes or edits are made and the original work is properly cited (including links to both the formal publication through the relevant DOI and the license). See: https://creativecommons.org/licenses/by-nc-nd/4.0/.

\section{References}

1. Lim IIP, Bondoc AJ, Geller JI, et al. Hepatoblastomathe evolution of biology, surgery, and transplantation. Children (Basel) 2018. doi: 10.3390/children6010001.

2. Bennett J, Kirby-Allen $\mathrm{M}, \mathrm{Ng}$ V, et al. Hepatoblastoma in a child with early-onset cirrhosis. J Pediatr Hematol Oncol 2019;41:e30-3.

3. Liu B, Cui X, Zheng S, et al. Aberrant KLK4 gene promoter hypomethylation in pediatric hepatoblastomas. Oncol Lett 2017;13:1360-4. 
4. Stafman LL, Williams AP, Garner EF, et al. Targeting PIM kinases affects maintenance of CD133 tumor cell population in hepatoblastoma. Transl Oncol 2019;12:200-8.

5. Tomlinson GE, Kappler R. Genetics and epigenetics of hepatoblastoma. Pediatr Blood Cancer 2012;59:785-92.

6. Qi P, Wang L, Zhou B, et al. Associations of miRNA polymorphisms and expression levels with breast cancer risk in the Chinese population. Genet Mol Res 2015;14:6289-96.

7. Arai Y, Honda S, Haruta M, et al. Genome-wide analysis of allelic imbalances reveals $4 \mathrm{q}$ deletions as a poor prognostic factor and MDM4 amplification at 1q32.1 in hepatoblastoma. Genes Chromosomes Cancer 2010;49:596-609.

8. Suzuki M, Kato M, Yuyan C, et al. Whole-genome profiling of chromosomal aberrations in hepatoblastoma using high-density single-nucleotide polymorphism genotyping microarrays. Cancer Sci 2008;99:564-70.

9. Sun L, Song L, Wan Q, et al. cMyc-mediated activation of serine biosynthesis pathway is critical for cancer progression under nutrient deprivation conditions. Cell Res 2015;25:429-44.

10. Takatsuno Y, Mimori K, Yamamoto K, et al. The rs6983267 SNP is associated with MYC transcription efficiency, which promotes progression and worsens prognosis of colorectal cancer. Ann Surg Oncol 2013;20:1395-402.

11. Chang J, Tian J, Yang Y, et al. A rare missense variant in TCF7L2 associates with colorectal cancer risk by interacting with a GWAS-identified regulatory variant in the MYC enhancer. Cancer Res 2018;78:5164-72.

12. Chang J, Tian J, Zhu Y, et al. Exome-wide analysis identifies three low-frequency missense variants associated with pancreatic cancer risk in Chinese populations. Nat Commun 2018;9:3688.

13. Chang J, Zhong R, Tian J, et al. Exome-wide analyses identify low-frequency variant in CYP26B1 and additional coding variants associated with esophageal squamous cell carcinoma. Nat Genet 2018;50:338-43.

14. Li J, Chang J, Tian J, et al. A rare variant P507L in TPP1 interrupts TPP1-TIN2 interaction, influences telomere length, and confers colorectal cancer risk in Chinese population. Cancer Epidemiol Biomarkers Prev 2018;27:1029-35.

15. He J, Wang F, Zhu J, et al. Association of potentially functional variants in the XPG gene with neuroblastoma risk in a Chinese population. J Cell Mol Med 2016;20:1481-90.

16. He J, Wang F, Zhu J, et al. The TP53 gene rs1042522
$\mathrm{C}>\mathrm{G}$ polymorphism and neuroblastoma risk in Chinese children. Aging (Albany NY) 2017;9:852-9.

17. He J, Zou Y, Wang T, et al. Genetic variations of GWASidentified genes and neuroblastoma susceptibility: a replication study in southern Chinese children. Transl Oncol 2017;10:936-41.

18. Stine ZE, Walton ZE, Altman BJ, et al. MYC, metabolism, and cancer. Cancer Discov 2015;5:1024-39.

19. Armengol C, Cairo S, Fabre M, et al. Wnt signaling and hepatocarcinogenesis: the hepatoblastoma model. Int J Biochem Cell Biol 2011;43:265-70.

20. Kress TR, Pellanda P, Pellegrinet L, et al. Identification of MYC-dependent transcriptional programs in oncogeneaddicted liver tumors. Cancer Res 2016;76:3463-72.

21. Hartwell HJ, Petrosky KY, Fox JG, et al. Prolactin prevents hepatocellular carcinoma by restricting innate immune activation of c-Myc in mice. Proc Natl Acad Sci U S A 2014;111:11455-60.

22. Han H, Sun D, Li W, et al. A c-Myc-microRNA functional feedback loop affects hepatocarcinogenesis. Hepatology 2013;57:2378-89.

23. Solé X, Hernández P, de Heredia ML, et al. Genetic and genomic analysis modeling of germline c-MYC overexpression and cancer susceptibility. BMC Genomics 2008;9:12.

24. Lee KE, Chang BC, Park S, et al. Effects of single nucleotide polymorphisms in c-Myc on stable warfarin doses in patients with cardiac valve replacements. Pharmacogenomics 2015;16:1101-8.

25. Franceschini N, Hu Y, Reiner AP, et al. Prospective associations of coronary heart disease loci in African Americans using the MetaboChip: the PAGE study. PLoS One 2014;9:e113203.

26. Wang H, Lu J, Edmunds LR, et al. Coordinated activities of multiple Myc-dependent and Myc-independent biosynthetic pathways in hepatoblastoma. J Biol Chem 2016;291:26241-51.

27. Purcell R, Childs M, Maibach R, et al. HGF/c-Met related activation of $\beta$-catenin in hepatoblastoma. J Exp Clin Cancer Res 2011;30:96.

28. Gammons M, Bienz M. Multiprotein complexes governing Wnt signal transduction. Curr Opin Cell Biol 2018;51:42-9.

Cite this article as: Yang T, Wen Y, Li J, Tan T, Yang J, Pan J, Hu C, Yao Y, Zhang J, Li S, Xia H, He J, Zou Y. Association of CMYC polymorphisms with hepatoblastoma risk. Transl Cancer Res 2020;9(2):849-855. doi: 10.21037/tcr.2019.12.19 


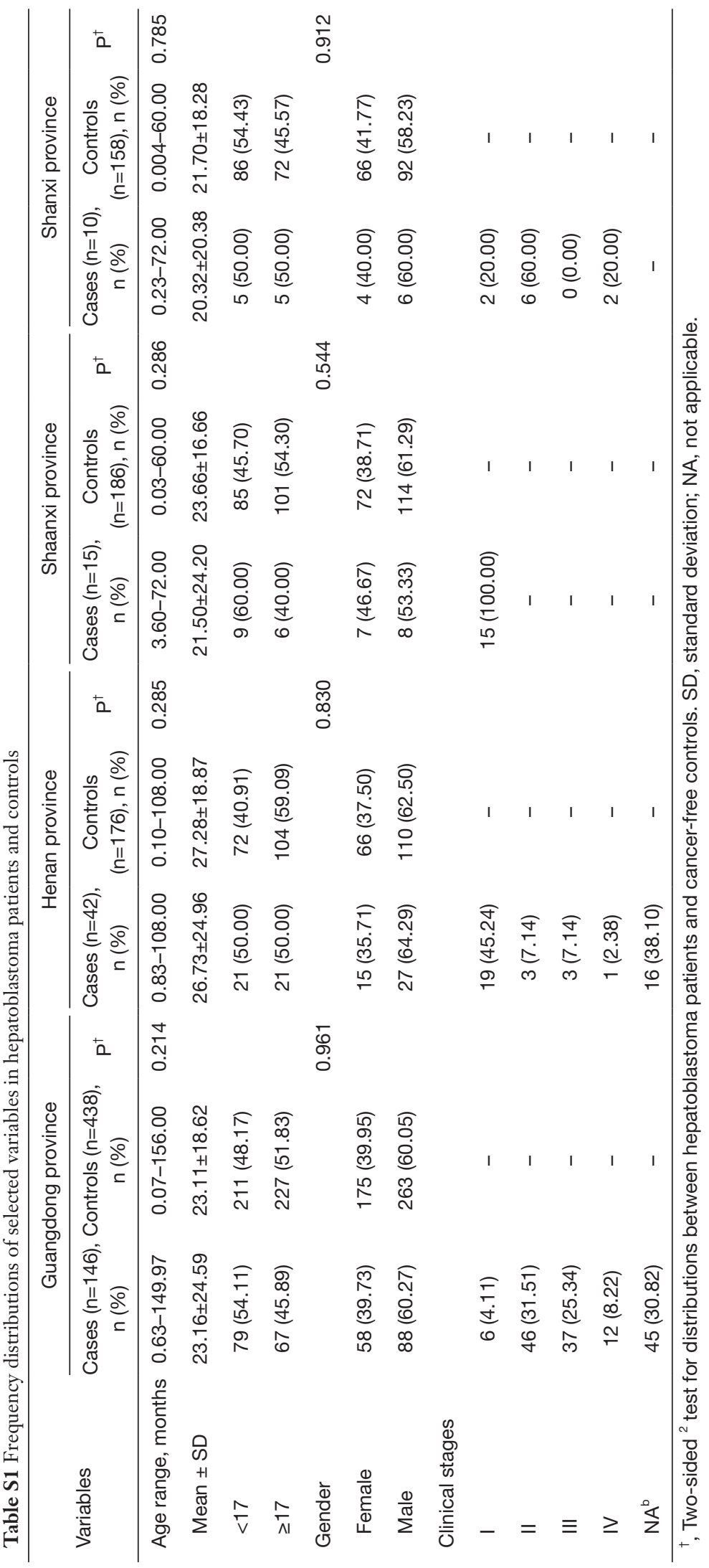

